\title{
New records of Leptopilina, Ganaspis, and Asobara species associated with Drosophila suzukii in North America, including detections of $L$. japonica and $\mathrm{G}$. brasiliensis
}

\author{
Paul K. Abram ${ }^{1,2,3}$, Audrey E. McPherson ${ }^{2}$, Robert Kula ${ }^{4}$, Tracy Hueppelsheuser ${ }^{5}$, \\ Jason Thiessen', Steve J. Perlman², Caitlin I. Curtis², Jessica L. Fraser'², \\ Jordan Tam ${ }^{3}$, Juli Carrillo ${ }^{3}$, Michael Gates ${ }^{4}$, Sonja Scheffer ${ }^{5}$, Matthew Lewis ${ }^{5}$, \\ Matthew Buffington ${ }^{5}$
}

I Agriculture and Agri-Food Canada, Agassiz Research and Development Centre, Agassiz, British Columbia, Canada 2 University of Victoria, Department of Biology, Victoria, British Columbia, Canada 3 The University

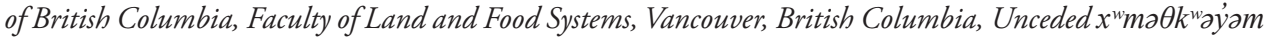
Musqueam Territory, Canada 4 Systematic Entomology Laboratory, USDA-ARS, clo National Museum of Natural History, Smithsonian Institution, Washington, D.C., USA 5 British Columbia Ministry of Agriculture, Plant Health Unit, Abbotsford, British Columbia, Canada

Corresponding author: Paul K. Abram (paul.abram@canada.ca)

Academic editor: Gavin Broad | Received 2 June 2020 | Accepted 24 July 2020 | Published 31 August 2020

http://zoobank.org/45F6DB8A-F429-4E2F-B863-D1A9049710AA

Citation: Abram PK, McPherson AE, Kula R, Hueppelsheuser T, Thiessen J, Perlman SJ, Curtis CI, Fraser JL, Tam J, Carrillo J, Gates M, Scheffer S, Lewis M, Buffington M (2020) New records of Leptopilina, Ganaspis, and Asobara species associated with Drosophila suzukii in North America, including detections of L. japonica and G. brasiliensis. Journal of Hymenoptera Research 78: 1-17. https://doi.org/10.3897/jhr.78.55026

\begin{abstract}
We report the presence of two Asian species of larval parasitoids of spotted wing Drosophila, Drosophila suzukii (Matsumura) (Diptera: Drosophilidae), in northwestern North America. Leptopilina japonica Novkovic \& Kimura and Ganaspis brasiliensis (Ihering) (Hymenoptera: Figitidae) were found foraging near and emerging from fruits infested by $D$. suzukii at several locations across coastal British Columbia, Canada in the summer and fall of 2019. While G. brasiliensis was found in British Columbia for the first time in 2019, re-inspection of previously collected specimens suggests that $L$. japonica has been present since at least 2016. Additionally, we found a species of Asobara associated with D. suzukii in British Columbia that is possibly Asobara rufescens (Förster) (known only from the Palearctic Region) based on COI DNA barcode data. These findings add to the list of cases documenting adventive es-
\end{abstract}

Copyright Paul K. Abram et al. This is an open access article distributed under the terms of the Creative Commons Attribution License (CC BY 4.0), which permits unrestricted use, distribution, and reproduction in any medium, provided the original author and source are credited. 
tablishment of candidate classical biological control agents outside of their native ranges. The findings also illustrate the need for revisiting species concepts within Asobara, as well as host and geographic distribution data due to cryptic and/or misidentified species.

\section{Keywords}

adventive establishment, Braconidae, classical biological control, Drosophilidae, Figitidae, Pteromalidae, spotted wing Drosophila

\section{Introduction}

Evidence is accumulating that increasing human activities are responsible for the redistribution of not only invasive pest insects but also many of their natural enemies (Roy et al. 2011; Mason et al. 2017; Weber et al. 2017). In the past few decades, there have been numerous cases where the arrival of invasive insect pests in their invaded range has been followed by the detection of closely associated parasitoids from their native range, which are assumed to be adventive; that is, accidentally introduced (Frewin et al. 2010; Mason et al. 2011, 2017; Talamas et al. 2015; Weber et al. 2017; Ganjisaffar et al. 2018; Stahl et al. 2019). In some cases, at the time of detection, these adventive parasitoids were under consideration for importation and intentional introduction against their pest hosts as classical biological control agents (Mason et al. 2011; Talamas et al. 2015; Abram et al. 2019). These new distribution records are made possible as a result of close collaboration between the biological control and taxonomic communities (Rosen 1986; Rosen and DeBach 1973; Talamas et al. 2015; Buffington et al. 2019).

Spotted wing Drosophila, Drosophila suzukii (Matsumura) (Diptera: Drosophilidae), is an invasive pest in North America, South America, and Europe that lays its eggs and completes its larval development inside the fruit of a large variety of crop and noncrop host plants (Lee at al. 2011; Asplen et al. 2015; Kenis et al. 2016). A few species of resident pupal parasitoids, including Pachycrepoideus vindemmiae Rondani (Hymenoptera: Pteromalidae), were found attacking $D$. suzukii in these invaded areas but parasitism levels were generally low (Lee et al. 2019). Larval parasitism of D. suzukii in invaded areas appears to be particularly uncommon, probably due to the inability of most genotypes of native larval parasitoids to overcome the immune defenses of $D$. suzukii (Lee et al. 2019; Kacsoh and Schlenke 2012; Poyet et al. 2013). Thus, soon after the introduction and spread of D. suzukii in invaded areas, exploration for potential classical biological control agents in China, South Korea, and Japan began with the goal of identifying parasitoid species that could be evaluated as potential classical biological control agents (Daane et al. 2016; Girod et al. 2018a; Giorgini et al. 2019). These surveys found several parasitoid species attacking D. suzukii larvae and pupae, but two larval parasitoids were responsible for the greatest levels of parasitism: Ganaspis brasiliensis (Ihering) and Leptopilina japonica Novkovic \& Kimura (Hymenoptera: Figitidae). These two species both parasitize early-instar larvae of D. suzukii inside fresh fruit (Wang et al. 2018), 
although L. japonica will also attack hosts in other substrates (e.g., banana traps) (Daane et al. 2016; Giorgini et al. 2019). Leptopilina japonica is known to attack Drosophilidae other than D. suzukii in the field in Asia (Novković et al. 2011). Ganaspis brasiliensis has only been reared from fresh fruits infested by D. suzukii and other closely related, microsympatric frugivorous Drosophilidae and thus appears to have a more restricted host range than L. japonica (Daane et al. 2016; Girod et al. 2018a; Giorgini et al. 2019). The host specificity of $G$. brasiliensis may also vary significantly among genetic lineages (Nomano et al. 2017; Girod et al. 2018b). Recently, G. brasiliensis was reared from sentinel traps containing larvae and pupae of Drosophila melanogaster Meigen (Diptera: Drosophilidae) in Mexico (Gonzales-Cabrera et al. 2020), representing the first time this species has been detected in continental North America.

Exploration for biological control agents of $D$. suzukii has also led to the discovery of described and several undescribed species of Asobara (Hymenoptera: Braconidae) attacking D. suzukii in Asia (Nomano et al. 2015; Guerrieri et al. 2016). Asobara japonica Belokobylskij was initially considered as a potential classical biological control agent of D. suzukii but was subsequently excluded from consideration due to its relatively broad host range (Daane et al. 2016; Girod et al. 2018b). Both Asian and European lines of another species in the same genus, Asobara tabida Nees, failed to develop on D. suzukii in laboratory experiments (Chabert et al. 2012; Nomano et al. 2015). North American Asobara species are not presently known to parasitize $D$. suzukii, although one species (putatively $A$. tabida) has been observed in a possible association with $D$. suzukii (Thistlewood et al. 2013).

In this article, we report the unexpected presence of both L. japonica and G. brasiliensis in northwest North America, representing the first time they have been detected in this area. These are the first records of G. brasiliensis in North America outside of Mexico, and the first records of $L$. japonica outside of its presumed native range of Asia. We also report one species of Asobara (Hymenoptera: Braconidae), as well as Pachycrepoideus vindemmiae (Hymenoptera: Pteromalidae), in probable association with $D$. suzukii.

\section{Materials and methods}

\section{Preliminary parasitoid collections}

On July 15, 2019, we first observed numerous parasitoids walking on and flying in the vicinity of raspberry (Rubus idaeus 'Rudi') fruits in an experimental plot heavily infested by D. suzukii in Agassiz, British Columbia, Canada (GPS: 49 $14^{\prime} 34.8^{\prime \prime N}$, $\left.121^{\circ} 45^{\prime} 18.0^{\prime \prime W}\right)$. Remarkably, we collected $>100$ parasitoid specimens by mouth aspirator in less than $45 \mathrm{~min}$ of effort by two individuals (PKA, personal observations). We also collected 20 raspberry fruits presumed to be infested by D. suzukii, which were divided between two ventilated plastic containers $(0.25 \mathrm{~L})$ with filter paper to observe whether any parasitoids successfully emerged. A subset of field-collected parasitoid individuals were exposed to $2^{\text {nd }}$ and $3^{\text {rd }}$-instar D. suzukii larvae (approx. 50 per vial) in 
two Drosophila rearing vials containing artificial diet (Formula 4-24 medium, Carolina Biological Supply Co. Burlington, NC) to informally test whether or not they were capable of successfully parasitizing D. suzukii larvae. Parasitoids emerged both from field-collected $D$. suzukii-infested raspberries and lab-reared D. suzukii larvae in artificial diet exposed to field-collected parasitoid adults (PKA, personal observations). This was surprising, given the known low developmental success of North American larval parasitoids attacking D. suzukii (Lee et al. 2019). This observation motivated an attempt to obtain a preliminary species-level identification. A 683 bp region of the cytochrome oxidase I (COI) gene of one aspirator-collected parasitoid specimen was barcoded and compared against existing records in GenBank using a nucleotide BLAST (NCBI, 2019). This sequence showed $98.67 \%$ identity with GenBank Accession AB546875.1, which is a sequence of L. japonica from Japan (Novkovic et al. 2016). Specimens from the same date and location that were collected by mouth aspirator $(\mathrm{n}=24)$, emerged from raspberry fruit $(\mathrm{n}=3)$, and that emerged from lab-reared $D$. suzukii larvae ( $\mathrm{n}=6$ ), were all identified as L. japonica (by MLB) based on morphology (see Identification of parasitoid specimens below for details on morphological determinations). This initial find prompted a re-examination of parasitoid specimens $(\mathrm{n}=14)$ emerging from 60 D. suzukii-infested strawberry (Fragaria $\times$ ananassa) fruits (total $\mathrm{n}$ = 375 emerging D. suzukii) collected near Langley, British Columbia in two commercial fields (GPS: $49^{\circ} 07^{\prime} 34.7^{\prime \prime} \mathrm{N}, 122^{\circ} 32^{\prime} 45.2^{\prime \prime} \mathrm{W}$ and $\left.49^{\circ} 08^{\prime} 07.8^{\prime \prime} \mathrm{N}, 122^{\circ} 33^{\prime} 42.1^{\prime \prime W}\right)$ between September 20 and 27, 2016. Based on morphology, the parasitoid specimens were also confirmed to be L. japonica, suggesting that this species has been present in British Columbia since at least 2016.

\section{Parasitoid collections throughout southwestern British Columbia}

Motivated by the unexpected detection of L. japonica in British Columbia, we next conducted additional collections in 2019 to determine how widespread in coastal British Columbia L. japonica might be, and whether any other native or exotic species of parasitoids might also be present. From August to October 2019, parasitoid specimens were obtained from 54 opportunistic collection samples from 23 different sites in coastal British Columbia (the lower mainland and Vancouver Island) (Fig. 1). Parasitoid specimens $(\mathrm{n}=525)$ were collected from plants and areas known to be infested by $D$. suzukii with one of three sampling methods: (1) mouth aspiration of parasitoids seen flying or resting on the foliage or fruits of Himalayan blackberry, Rubus armeniacus ( $\mathrm{n}=34$ samples) or cultivated blackberry, Rubus fruticosis 'Triple Crown' ( $\mathrm{n}=$ 2 samples); (2) collection of $R$. armeniacus fruits (approx. 20-60 berries per sample) into ventilated plastic rearing containers (volume: 0.5 or $1.0 \mathrm{~L}$ ) lined with filter paper to rear out parasitoids and host Drosophilidae in the laboratory (16:8 hour light:dark, $\left.24 \pm 1{ }^{\circ} \mathrm{C}\right)(\mathrm{n}=14) ;(3)$ separation of parasitoid by-catch from plastic traps baited with apple cider vinegar placed in hedgerows on a university experimental farm to collect $D$. suzukii ( $\mathrm{n}=2$ ); and (4) collection of $R$. armeniacus berries followed by exposure outside 
in a plastic trap for 6 days, and then rearing out insects in glass jars in the laboratory $(\mathrm{n}=2)$. Collections from $R$. armeniacus were in suburban or natural areas along roadways or in parks. For a subset of $14 R$. armeniacus fruit collections from which at least one parasitoid emerged, emerging Drosophilidae $(\mathrm{n}=1,637)$ were also counted and identified. All identifiable emerged Drosophilidae were either D. suzukii (85.8 \%) or D. melanogaster $(0.6 \%)$. Remaining flies $(13.6 \%)$ could not be identified conclusively due to deterioration or mold in containers. Full information on collection sites, dates, and methods, and insect emergence if applicable, can be found in the Suppl. material 1. Due to the opportunistic nature of our sampling in space and time, and unknown levels of parasitoid and host mortality in rearing, we did not calculate or present percent parasitism levels. All collected parasitoids were preserved in $95 \% \mathrm{EtOH}$ for subsequent morphological and molecular identification.

\section{Identification of parasitoid specimens}

Specimens from both initial and more extensive collections in British Columbia were sent to MLB after initial COI barcoding (by SJP and CC) yielded results consistent with barcode sequences on GenBank under the name L. japonica (see above). Upon further examination, it was determined that not only was $L$. japonica present in the samples but so too were $G$. brasiliensis, an undetermined species of Asobara, and $P$. vindemmiae (Figs 2-7). Specimens of L. japonica and G. brasiliensis were vacuum dried and card mounted; the Asobara and Pachycrepoideus specimens were prepared using HMDS (Heraty and Hawks 1998) and point mounted. Specimens are deposited in the insect collection of the National Museum of Natural History (USNM; Smithsonian Institution, Washington DC) and the Canadian National Collection of Insects, Arachnids, and Nematodes (Ottawa, Ontario, Canada).

The morphology-based research in Novković et al. (2011), Buffington and Forshage (2016) and Lue et al. (2016), as well as specimens in the USNM, were used to confirm the identities of the Figitidae. While this research was being conducted, it became clear that specimens of Leptopilina submitted to MLB for identification in 2016 were misidentified as Leptopilina heterotoma (Thompson). At that time, the USNM lacked specimens of L. japonica, making a direct comparison impossible. Recently, the USNM has received voucher specimens from the original paper describing L. japonica, as well as additional material from China and Korea. This made direct comparisons possible, and the identity of the 2016 specimens was corrected.

In terms of Figitidae attacking D. suzukii, L. japonica and G. brasiliensis can be difficult to distinguish (Figs 2, 3). Careful examination of the posterior aspect of the metapleuron reveals a complete patch of setae in G. brasiliensis (Fig. 4a), while the same location in L. japonica is glabrous (Fig. 5a). Additionally, the metasoma setal hair ring is complete in G. brasiliensis (Fig. $4 \mathrm{~b}$ ) and dorsally incomplete in L. japonica (Fig. 5b). Lastly, the metapleuron, in lateral view, is simple in G. brasiliensis (Fig. 4c); in L. japonica, a longitudinal carina transverses the middle of the metapleuron (Fig. 5c). 
Specimens of Asobara, Pachycrepoideus, and Figitidae are relatively easy to differentiate from one another given their overall dissimilar shape and color (Figs 6, 7).

The single Pteromalidae specimen was identified and confirmed using Bouček and Heydon (1997) and by comparison with authoritatively identified specimens in the USNM. The specimens of Asobara were sight identified to genus; Wharton (1980), Vet et al. (1984), and Nomano et al. (2015) were consulted in an effort to identify the specimens to species.

A subset of the Figitidae and Braconidae specimens were sequenced for the COI 'barcode' region; as only one Pachycrepoideus was collected, no barcode was generated for this species. DNA was extracted using the QIAamp DNA Micro Kit (Qiagen). PCR amplifications of COI were carried out using LCO1490 (Folmer et al. 1994) and C1-N-2191 (Simon et al. 1994) with a Bio-Rad T100 thermal cycler under the following cycling conditions: initial denaturation for $7 \mathrm{~min}$ at $95^{\circ} \mathrm{C}, 35$ cycles of $30 \mathrm{~s}$ at $95^{\circ} \mathrm{C}, 30 \mathrm{~s}$ at $50{ }^{\circ} \mathrm{C}, 45 \mathrm{~s}$ at $72{ }^{\circ} \mathrm{C}$, and a final extension for $1.5 \mathrm{~min}$ at $72{ }^{\circ} \mathrm{C}$. PCR products were cleaned with ExoSAP-IT (Affymetrix), sequenced with BigDye v.3.1 (Life Technologies) and run on an ABI 3730xl automated DNA sequencer (Applied Biosystems). Contigs were assembled and edited in Geneious Prime 2020.04 (Biomatters). Sequences used in the study have been deposited in GenBank (Accession nos. MT559416-MT559426).

\section{Results and discussion}

\section{Figitidae}

Leptopilina japonica was the most common parasitoid species found in our collections, representing $95.8 \%$ (503/525) of all parasitoids we collected and identified in British Columbia in 2019. This species was present across the sampled area (Fig. 1) and was collected with all three sampling methods (traps, aspirator collections, rearing from infested fruit). Ganaspis brasiliensis was the next most common species $(2.7 \%$ of all specimens) and was present at three sites, which were all in the eastern part of the sampled geographic range (Fig. 1). This species was collected both with aspirators while foraging on berries and emerging from D. suzukii-infested berries. Somewhat surprisingly, no native Leptopilina spp. were found in our samples in 2019.

Results from DNA barcoding of a small subset of Figitidae specimens was consistent with all morphologically-based identifications. COI sequences from L. japonica showed high similarity to specimens previously collected in China and Japan (> $98 \%$ identity). All four of the $G$. brasiliensis specimens from British Columbia that we barcoded clustered within the "G1" clade (100\% identity), which has previously been found in South Korea, China, and Japan (Nomano et al. 2017; Giorgini et al. 2019) and appears to be the most host-specific lineage of $G$. brasiliensis (Nomano et al. 2017; Girod et al. 2018b). Further studies are needed to determine the levels of genetic diversity of $L$. japonica and $G$. brasiliensis in North America and potential source populations of these parasitoids in Asia. 


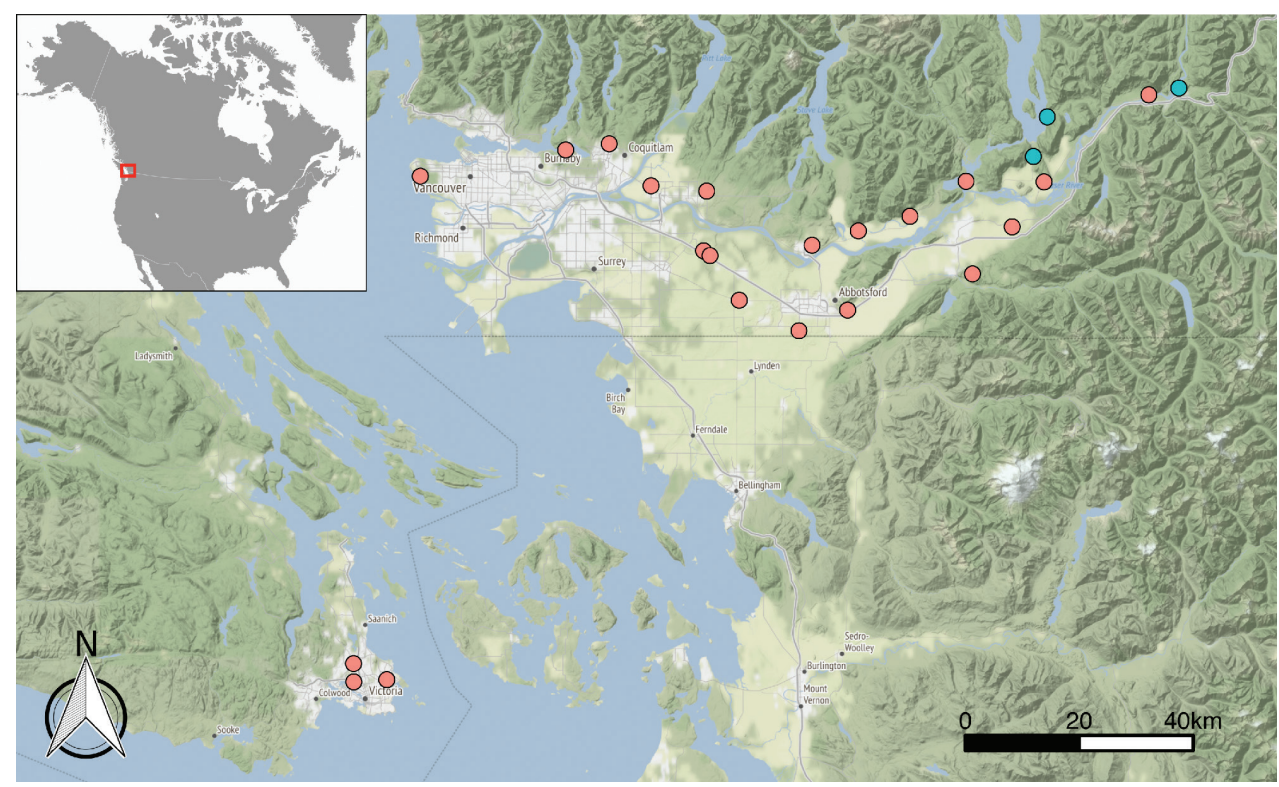

Figure I. Map of sites where Leptopilina japonica (red circles), or both L. japonica and Ganaspis brasiliensis (green circles) were found in British Columbia, Canada in 2016 and 2019. The red box in the inset shows where the mapped area is situated in North America. Map tiles by Stamen Design, under CC BY 3.0. Data by OpenStreetMap, under ODbL.

We emphasize that because our sampling took place within a restricted time window and set of habitats, our data are likely not representative of the two adventive figitid parasitoid species' relative abundances or impact. They simply demonstrate that these two species are present in the south coastal region of British Columbia. More widespread, season-long surveys in several habitats are needed to determine the relative abundance and distribution of each species. Similarly, repeated fruit collections containing known life stage distributions of hosts, coupled with population modelling approaches such as stage-structured matrix models (e.g., Wiman et al. 2014, 2016), will be necessary to estimate the population-level impact of these adventive exotic parasitoids attacking $D$. suzukii - and possibly other, non-target Drosophilidae - in North America.

\section{Braconidae}

One species of Asobara ( $1.5 \%$ of all parasitoids in collections) was collected with aspirators from berries $(\mathrm{n}=3)$ and reared from $D$. suzukii-infested blackberries $(\mathrm{n}=$ 5) from two sites. Although nearly all of the Drosophilidae emerging from these collections were D. suzukii, we cannot exclude the possibility that the five Asobara that emerged from berries could have been associated with $D$. melanogaster, which was present at very low levels in our samples (see above). Exposure of three female Asobara to lab-reared D. suzukii larvae in artificial diet (see methods in "Preliminary parasitoid 


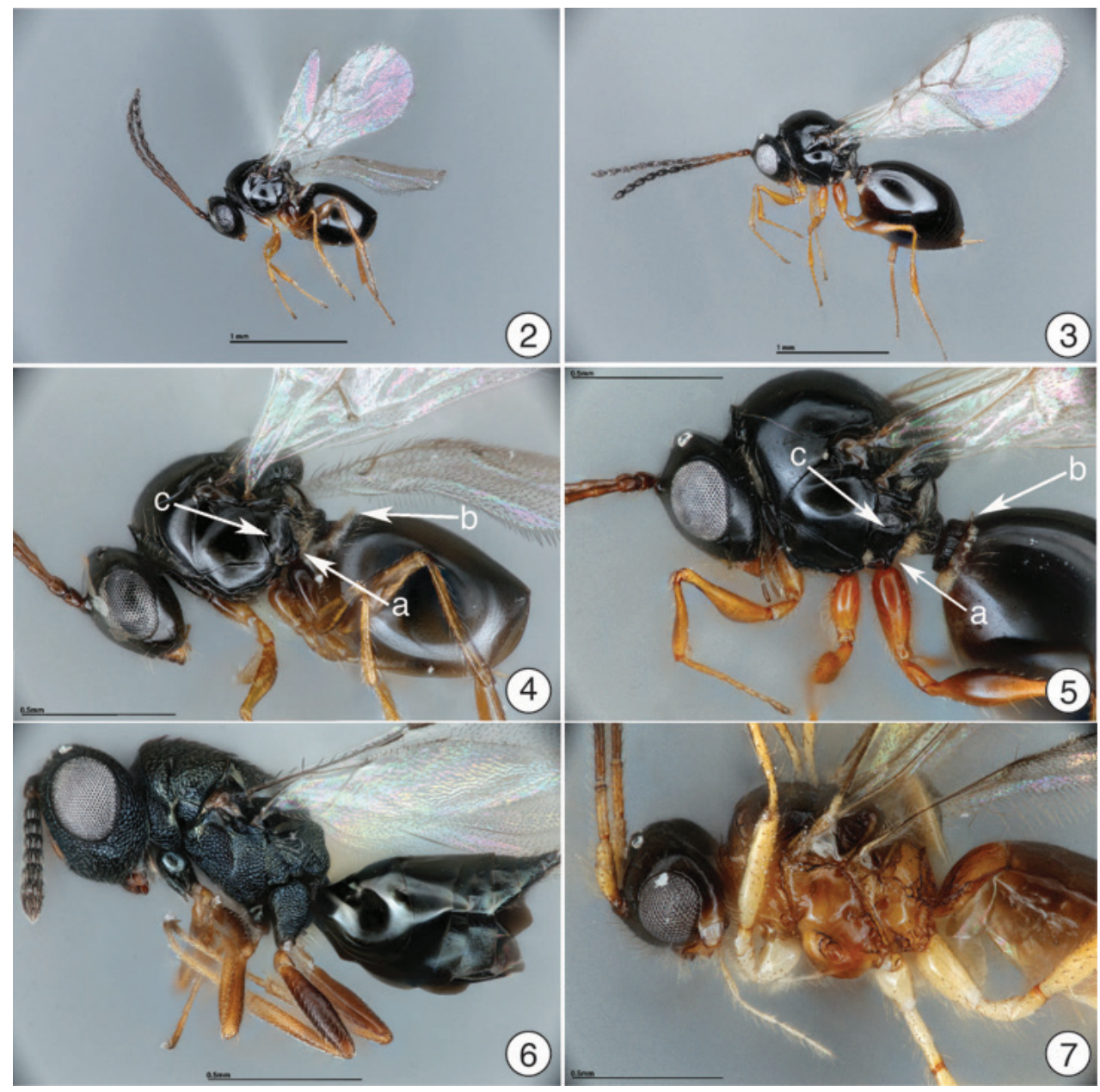

Figures 2-7. Ganaspis brasiliensis (2, 4); Leptopilina japonica (3, 5); Pachycrepoideus vindemmiae (6); Asobara sp. (7).

collections" above) only yielded a single male offspring (PKA, personal observations). While the field collections and single lab observation provides a proof-of-concept that this Asobara species can attack and develop in D. suzukii, at this time the association of this Asobara species with D. suzukii should be considered tentative. As far as is known, species of Asobara are solitary koinobiont endoparasitoids of cyclorrhaphous flies, mostly Drosophilidae (Wharton 1997, Yu et al. 2016).

Two species of Asobara, A. tabida (Nees) and A. fungicola (Ashmead), are known from the Nearctic Region, and both species have been reported from British Columbia (Yu et al. 2016). One of us (RRK) initially identified the Asobara we found associated with D. suzukii in British Columbia as A. tabida using Wharton (1980), but A. tabida and A. fungicola are the only species included in the key. Several species of Asobara, including A. tabida and A. fungicola, occur in multiple zoogeographic regions (Yu et al. 
2016). Therefore, it is possible that other Asobara species morphologically similar to $A$. tabida and $A$. fungicola occur in the Nearctic Region but are commonly misidentified as the aforementioned species. For example, Asobara rufescens (Förster) and A. tabida are extremely similar morphologically; Vet et al. (1984) considered them sibling species and identified subtle differences in color, shape of antennal flagellomeres 1 and 2, tarsal claw length, and the basitarsus. Asobara rufescens (Förster, 1863) is currently known only from the Palearctic Region but has been considered a junior synonym of A. tabida (Nees, 1834), which occurs in the Nearctic, Palearctic, Oriental, and Oceanic regions. Thus, if $A$. rufescens occurs in the Nearctic Region it could be easily misidentified as $A$. tabida, especially when using identification tools that were based on region or published during the period of time when $A$. rufescens was a junior synonym of A. tabida. Recent efforts to accurately identify species of Asobara have relied on COI DNA barcoding along with authoritative identifications based on morphological features (Nomano et al. 2015, Guerrieri et al. 2016).

We obtained a 658-bp fragment of the COI DNA barcoding region from one of the specimens RRK tentatively identified as A. tabida. A GenBank nucleotide BLAST search (NCBI 2019) using the 658-bp fragment, as well as a search of all COI barcode records using the Identification System in the Barcode of Life Database (BOLD; Ratnasingham and Hebert 2007), yielded 53 COI sequences we consider conspecific with our Asobara specimen. Of the 41 sequences from GenBank, 39 had 98.79-100\% identity (79-100\% query coverage) with the Asobara COI sequence obtained in this research; percent identities for the two most dissimilar sequences that we still consider conspecific were $97.24 \%$ and $97.26 \%$ (100\% and $93 \%$ query coverage, respectively). The 41 sequences from GenBank were mostly from specimens identified as $A$. rufescens $(\mathrm{n}=36)$ but also included some identified only as Braconidae or Hymenoptera $(n=5)$. The sequences in BOLD consisted of the 41 recovered via GenBank and an additional 12 sequences we consider conspecific with our Asobara species. Of the 53 sequences recovered, 51 have sequence similarity ranging from $99.18-100 \%$, and two have sequence similarity ranging from 97.19-97.22\%; all are in BOLD under the name $A$. rufescens. One sequence in both GenBank and BOLD under the name $A$. rufescens (GenBank: KT604569.1) appeared to have quality control issues and was not considered in this research. The most similar sequence in GenBank and BOLD that we do not consider conspecific was from a specimen identified as A. tabida, with $93.46 \%$ identity (99\% query coverage) in GenBank and $93.36 \%$ similarity in BOLD relative to our Asobara sequence.

Sequences in BOLD/GenBank identified as A. rufescens were likely determined as such based solely on their high similarity to sequences already in those databases from specimens identified as $A$. rufescens using morphological features. Notably, COI sequences from putative $A$. rufescens specimens from Japan (Nomano et al. 2015; GenBank: AB920758.1, AB920759.1, AB920763.1, AB920762.1), identified by M. Kimura using the morphological features from Vet et al. (1984), are likely the basis for all subsequent COI sequence identifications in BOLD/GenBank. If the specimens from Japan are accurately identified as $A$. rufescens, a specimen of $A$. rufescens collected 
in Puslinch, Ontario, Canada on August 14, 2008 (Hebert et al. 2016; GenBank: KR884537.1) would be the first record of A. rufescens in the Western Hemisphere. Further, collection data for all sequences in BOLD/GenBank putatively conspecific with M. Kimura's $A$. rufescens reflect that this species was collected in Canada every year from 2008-2019 (except 2011) with occurrences ranging from Forillon National Park, Quebec (Hebert et al. 2016; GenBank: KR420364.1) to Elk Island National Park, Alberta (Hebert et al. 2016; GenBank: KR793444.1). Thus, A. rufescens has possibly occurred in North America since at least 2008, the year D. suzukii was first detected on the continent (Asplen et al. 2015), and ranges from east to west across nearly all of Canada. Thistlewood et al. (2013) reported specimens of Asobara reared from fermenting banana that was artificially infested with $D$. suzukii and then placed in the field in British Columbia, which they considered likely to be $A$. tabida. The Asobara specimens reported in Thistlewood et al. (2013) might be conspecific with our Asobara species from British Columbia. If so, it is possible that our Asobara species (possibly A. rufescens) has been associated with $D$. suzukii since at least 2011; however, this is uncertain regardless of Asobara species identity because the banana baits were infested with Drosophilidae other than D. suzukii after being placed in the field.

Kimura's identification of $A$. rufescens from Japan, based on the morphological features identified in Vet et al. (1984) for differentiating A. rufescens and $A$. tabida (Nomano et al. 2015, M. Kimura personal communication), should be considered tentative. Features used by Vet et al. (1984) lack enough detail to reliably differentiate species of Asobara that are, perhaps, morphologically cryptic (R. Kula personal observation). The features used were differences, "in the shape of the $3^{\text {rd }}$ and $4^{\text {th }}$ antennal segment... and in the relative length of the claws," as well as unspecified differences "in the colour" and "in the basitarsus of the hind legs" (Vet et al. 1984). These features might reflect differences between $A$. rufescens and $A$. tabida, but accurate interpretation is difficult because the color differences were not illustrated or specified, the basitarsus difference was not elaborated or quantified, and the shape and length differences in the flagellomeres and tarsal claws, respectively, were not quantified. Line drawings were used to illustrate the relevant portions of the antenna and tarsus for specimens Vet et al. (1984) considered A. rufescens and $A$. tabida, as well as an $A$. rufescens $\times A$. tabida hybrid; thus, identification requires interpreting subtle differences in the line drawings by eye and without consideration for potential intraspecific variation. Thus, the features identified in Vet et al. (1984) require additional assessment to determine their utility.

Beyond morphological features, Vet et al. (1984) discerned via olfactometer tests that what they initially considered $A$. tabida actually consisted of two species attracted to different host habitats. One of the species was attracted to the odor of fermenting fruit, while the other species was attracted to the odor of decaying leaves, thus resulting in a premating reproductive barrier. The authors discerned, through comparison of their Asobara specimens with the holotype of $A$. rufescens for aforementioned morphological features, that the specimens attracted to decaying leaves were $A$. rufescens. Thus, the authors regarded $A$. tabida as a parasitoid of frugivorous Drosophilidae and $A$. rufescens as a parasitoid of saprophagous Drosophilidae. Mitsui et al. (2007) reported 
A. tabida and A. rufescens from Drosophilidae, including the former from D. suzukii, infesting clumps of banana placed at field sites; they also reported A. rufescens from Scaptomyza pallida (Zetterstedt) in decaying leaves. However, Chabert et al. (2012) found that $A$. tabida rarely oviposits into $D$. suzukii. Other research has shown, based on analysis of COI DNA barcoding data, that other Asobara specimens perceived as conspecific are apparently complexes of morphologically similar species (Nomano et al. 2015, Guerrieri et al. 2016) with different patterns of host use. Thus, additional research is needed to discern the limits of species in Asobara, as well as patterns of host use and factors that affect those patterns.

The COI sequence from our Asobara species from British Columbia was also very similar to COI sequences from putative A. rufescens collected in Germany (BOLD: 99.28-99.53\% similarity; Matthias et al. 2016) and Japan (GenBank: 99.20-99.24\% identity, 94-100 \% query coverage; BOLD: 99.18-99.23\% similarity; Nomano et al. 2015). Asobara rufescens has been collected in the Palearctic Region from Japan to the Netherlands (Yu et al. 2016); thus, if the identifications in GenBank and BOLD are accurate, $A$. rufescens is distributed broadly across the entire Holarctic Region. Conversely, while $A$. tabida has been reported across the entire Palearctic Region, from Japan and Russia (i.e., Sakhalin Oblast) west to Ireland, it has been reported infrequently in North America (Wharton 1980, Kraaijeveld and van der Wel 1994, Hoang 2002). Interestingly, there are no COI DNA barcode sequences in either GenBank or BOLD from specimens of $A$. tabida in North America. This raises the possibility that specimens in North America identified previously as A. tabida are actually $A$. rufescens. The results reported herein demonstrate the need for discerning species complexes within Asobara overall and its implications for interpreting patterns of host use. It would also be useful to sequence nuclear genes in Asobara, as some of the confusion in resolving $A$. tabida and $A$. rufescens may be due to hybridization and mitochondrial introgression.

\section{Pteromalidae}

Only a single specimen of $P$. vindemmiae was found in our collections. Pachycrepoideus vindemmiae is an idiobiont ectoparasitoid recorded as a primary parasitoid of Diptera, Lepidoptera, Hemiptera, and Hymenoptera. Diptera account for most records with 13 families, 33 genera, and 55 species recorded (Noyes 2019). As a facultative hyperparasitoid, hosts include Diptera (Sarcophagidae, Tachinidae) and Hymenoptera (Braconidae, Diapriidae, Encyrtidae, Figitidae, Eulophidae, Pteromalidae). Various factors influence the degree of primary vs. secondary parasitism in Drosophila systems (Bezerra Da Silva et al. 2019, Chen et al. 2015, Goubault et al. 2003, Rossi Stacconi et al. 2015, Wang et al. 2016). Related to the work herein, P. vindemmiae is known as a parasitoid of D. melanogaster, A. tabida and L. heterotoma (Noyes 2019, Philips 1993, Van Alphen and Thunnissen 1982), and also parasitizes conspecifics (Chen et al. 2015). Future studies could investigate whether $P$. vindemmiae could act as a facultative hyperparasitoid of L. japonica and G. brasiliensis in North America. 


\section{Conclusions}

Our findings here add to the growing list of recent high-profile invasive insect pests whose closely associated natural enemies have established adventive populations on new continents (Frewin et al. 2010; Talamas et al. 2015; Mason et al. 2017; Weber et al. 2017; Ganjisaffar et al. 2018; Stahl et al. 2019). Given that we found L. japonica to be remarkably common and widespread in our study area, it is quite possible that this species has also established in other areas outside of Asia but has not been detected yet. Ganaspis brasiliensis was much less common and widespread in our collections, and until more extensive sampling throughout the entire season is done, we cannot conclude whether or not it is well established in British Columbia. The Asobara species (potentially $A$. rufescens) we collected in probable association with $D$. suzukii also merits further investigation, both in terms of its systematics in relation to other Asobara worldwide and the extent of its potential impact on $D$. suzukii. We encourage researchers across North America, South America, and Europe to re-evaluate whether these parasitoids may be attacking D. suzukii in other invaded areas.

\section{Acknowledgements}

We thank Peggy Clarke, Matt Walz, Chris Hou, Sasha Tuttle, Nemo DeJong, Chandra Moffat, Mairi Robertson, Jade Sherwood, Warren Wong, and Yonathan Uriel for assistance in the laboratory and field. We also thank Markus Clodius for offering access to raspberry and blackberry plots. We thank Marc Kenis, Tim Haye, and Lukas Seehausen for providing specimens of $L$. japonica previously collected in China. This research was supported by funding from Agriculture and Agri-Food Canada (PKA and JC), the Canadian Agriculture Partnership Organic Science Cluster (PKA and JC), and Genome British Columbia (PKA and SJP). Mention of trade names or commercial products in this publication is solely for the purpose of providing specific information and does not imply recommendation or endorsement by the USDA. USDA is an equal opportunity provider and employer.

\section{References}

Abram PK, Talamas EJ, Acheampong S, Mason PG, Gariepy TD (2019) First detection of the samurai wasp, Trissolcus japonicus (Ashmead) (Hymenoptera, Scelionidae), in Canada. Journal of Hymenoptera Research 68: 29-36. https://doi.org/10.3897/jhr.68.32203

Asplen MK, Anfora G, Biondi A, Choi D-S, Chu D, Daane KM, Gibert P, Gutierrez AP, Hoelmer KA, Hutchison WD, Isaacs R, Jiang Z-L, Kárpáti Z, Kimura MT, Pascual M, Philips CR, Plantamp C, Ponti L, Vétek G, Vogt H, Walton VM, Yu Y, Zappalà L, Desneux N (2015) Invasion biology of spotted wing Drosophila (Drosophila suzukii): a global perspective and future priorities. Journal of Pest Science 88: 469-494. https://doi.org/10.1007/s10340-015-0681-z 
Bezerra Da Silva CS, Price BE, Soohoo-Hui A, Walton VM (2019) Factors affecting the biology of Pachycrepoideus vindemmiae (Hymenoptera: Pteromalidae), a parasitoid of spotted-wing drosophila (Drosophila suzukii). PLoS ONE 14: e0218301. https://doi.org/10.1371/journal.pone.0218301

Bouček Z, Heydon S (1997) Chapter 17 - Pteromalidae. In: Gibson GA, Huber JT, Woolley B (Eds) Annotated keys to the genera of Nearctic Chalcidoidea (Hymenoptera). NRC Research Press, 794 pp.

Buffington ML, Forshage M (2016) Redescription of Ganaspis brasiliensis (Ihering, 1905), new combination, (Hymenoptera: Figitidae) a natural enemy of the invasive Drosophila suzukii (Matsumura, 1931) (Diptera: Drosophilidae). Proceedings of the Entomological Society of Washington 118:1-13. https://doi.org/10.4289/0013-8797.118.1.1

Buffington ML, Talamas ET, Hoelmer KA (2019) Team Trissolcus: integrating taxonomy and biological control to combat the brown marmorated stink bug. American. Entomologist 64: 224-232. https://doi.org/10.1093/ae/tmy057

Chabert S, Allemand R, Poyet M, Eslin P, Gibert P (2012) Ability of European parasitoids (Hymenoptera) to control a new invasive Asiatic pest, Drosophila suzukii. Biological Control 63: 40-47. https://doi.org/10.1016/j.biocontrol.2012.05.005

Chen W, He Z, Ji X-L, Tang S-T, Hu H-Y (2015) Hyperparasitism in a generalist ectoparasitic pupal parasitoid, Pachycrepoideus vindemmiae (Hymenoptera: Pteromalidae), on its own conspecifics: when the lack of resource lead to cannibalism. PLoS ONE 10(4): e0124305. https://doi.org/10.1371/journal.pone.0124305

Daane KM, Wang X-G, Biondi A, Miller B, Miller JC, Riedl H, Shearer PW, Guerrieri E, Giorgini M, Buffington M, van Achterberg K, Song Y, Kang T, Yi H, Jung C, Lee DW, Chung B-K, Hoelmer KA, Walton VM (2016) First exploration of parasitoids of Drosophila suzukii in South Korea as potential classical biological agents. Journal of Pest Science 89:823-835. https://doi.org/10.1007/s10340-016-0740-0

Folmer O, Black M, Hoeh W, Lutz R, Vrijenhoek R (1994) DNA primers for amplification of mitochondrial cytochrome c oxidase subunit I from diverse metazoan invertebrates. Molecular Marine Biology and Biotechnology 3: 294-297.

Förster A ([1862]1863) Synopsis der Familien und Gattungen der Braconen - Verhandlungen des Naturhistorischen Vereines preussischen Rheinlande und Westphalens. 19: 225-288.

Frewin AJ, Xue Y, Welsman JA, Broadbent BA, Schaafsma AW, Hallett RH (2010) Development and parasitism by Aphelinus certus (Hymenoptera: Aphelinidae), a parasitoid of Aphis glycines (Hemiptera: Aphididae). Environmental Entomology 39: 1570-1578. https://doi. org/10.1603/EN09312

Ganjisaffar F, Talamas EJ, Bon MC, Gonzalez L, Brown BV, Perring TM (2018) Trissolcus hyalinipennis Rajmohana \& Narendran (Hymenoptera, Scelionidae), a parasitoid of Bagrada hilaris (Burmeister) (Hemiptera, Pentatomidae), emerges in North America. Journal of Hymenoptera Research 65: 111-130. https://doi.org/10.3897/jhr.65.25620

Geiger M, Moriniere J, Hausmann A, Haszprunar G, Wägele W, Hebert P, Rulik B (2016) Testing the global malaise trap program - How well does the current barcode reference library identify flying insects in Germany? Biodiversity Data Journal 4: e10671. https:// doi.org/10.3897/BDJ.4.e10671 
Giorgini M, Wang X-G, Wang Y, Chen F-S, Hougardy E, Zhang H-M, Chen Z-Q, Chen H-Y, Liu C-X, Cascone P, Formisano G, Carvalho GA, Biondi A, Buffington M, Daane KM, Hoelmer KA, Guerrieri E (2019) Exploration for native parasitoids of Drosophila suzukii in China reveals a diversity of parasitoid species and narrow host range of the dominant parasitoid. Journal of Pest Science 92: 509-522. https://doi.org/10.1007/s10340-018-01068-3

Girod P, Borowiec N, Buffington M, Chen G, Fang Y, Kimura MT, Peris-Felipo FJ, Ris N, Wu H, Xiao C, Zhang J, Aebi A, Haye T, Kenis M (2018a) The parasitoid complex of D. suzukii and other fruit feeding Drosophila species in Asia. Scientific Reports 8: 11839. https://doi.org/10.1038/s41598-018-29555-8

Girod P, Lierhmann O, Urvois T, Turlings TCJ, Kenis M, Haye T (2018b) Host specificity of Asian parasitoids for potential classical biological control of Drosophila suzukii. Journal of Pest Science 91: 1241-1250. https://doi.org/10.1007/s10340-018-1003-z

Gonzalez-Cabrera J, Cordoba-Urtiz EG, Moreno-Carrillo G, Sanchez-Gonzalez JA, Arredondo-Bernal HC (2020). First report of the parasitoid Ganaspis brasiliensis Ihering (Hymenoptera: Figitidae) in Mexico. Entomological News 129: 67-70. https://doi. org/10.3157/021.129.0110

Goubault M, Plantegenest M, Poinsot D, Cortesero AM (2003) Effect of expected offspring survival probability on host selection in a solitary parasitoid. Entomologia Experimentalis et Applicata 109: 123-131. https://doi.org/10.1046/j.1570-7458.2003.00101.x

Guerrieri E, Giorgini M, Cascone P, Carpenito S, van Achterberg C (2016) Species diversity in the parasitoid genus Asobara (Hymenoptera: Braconidae) from the native area of the fruit fly pest Drosophila suzukii (Diptera: Drosophilidae). PLoS One 11: e0147382. https://doi. org/10.1371/journal.pone.0147382

Hebert PDN, Ratnasingham S, Zakharov EV, Telfer AC, Levesque-Beaudin V, Milton MA, Pedersen S, Jannetta P, deWaard JR (2016) Counting animal species with DNA barcodes: Canadian insects. Philosophical Transaction of the Royal Society B 371: 20150333. https://doi.org/10.1098/rstb.2015.0333

Heraty J, Hawks D (1998) Hexamethyldisilazane - a chemical alternative for drying insects. Entomological News 109: 369-374.

Hoang A (2002) Physiological consequences of immune response by Drosophila melanogaster (Diptera: Drosophilidae) against the parasitoid Asobara tabida (Hymenoptera: Braconidae). Journal of Evolutionary Biology 15: 537-543. https://doi.org/10.1046/j.14209101.2002.00426.x

Kacsoh BZ, Schlenke TA (2012) High hemocyte load is associated with increased resistance against parasitoids in Drosophila suzukii, a relative of D. melanogaster. PLoS ONE 7: e34721. https://doi.org/10.1371/journal.pone.0034721

Kenis M, Tonina L, Eschen R, van der Sluis B, Sancassani M, Mori N, Haye T, Helsen H (2016) Non-crop plants used as hosts by Drosophila suzukii in Europe. Journal of Pest Science 89: 735-748. https://doi.org/10.1007/s10340-016-0755-6

Kraaijeveld AR, van der Wel NN (1994) Geographic variation in reproductive success of the parasitoid Asobara tabida in larvae of several Drosophila species. Ecological Entomology 19: 221-229. https://doi.org/10.1111/j.1365-2311.1994.tb00413.x 
Lee JC, Bruck DJ, Dreves AJ, Ioriatti C, Vogt H, Baufeld P (2011) In focus: spotted wing drosophila, Drosophila suzukii, across perspectives. Pest Management Science 67: 1349-1351. https://doi.org/10.1002/ps.2271

Lee JC, Wang X, Daane KM, Hoelmer KA, Isaacs R, Sial AA, Walton VM (2019) Biological control of spotted-wing Drosophila (Diptera: Drosophilidae) - current and pending tactics. Journal of Integrated Pest Management 10: 13. https://doi.org/10.1093/jipm/pmz012

Lue C-H, Driskell AC, Leips J, Buffington ML (2016) Review of the genus Leptopilina (Hymenoptera, Cynipoidea, Figitidae, Eucoilinae) from the Eastern United States, including three newly described species. Journal of Hymenoptera Research 53: 35-76. https://doi. org/10.3897/jhr.53.10369

Mason PG, Miall JH, Bouchard P, Gillespie DR, Broadbent AB, Gibson GAP (2011) The parasitoid communities associated with an invasive canola pest, Ceutorhynchus obstrictus (Coleoptera: Curculionidae), in Ontario and Quebec, Canada. Canadian Entomologist 143: 524-537. https://doi.org/10.4039/n11-041

Mason PG, Olfert OO, Haye T, Gariepy TD, Abram PK, Gillespie DR (2017) Risks and benefits of accidental introductions of biological control agents in Canada. In: Proceedings of the $5^{\text {th }}$ International Symposium on Biological Control of Arthropods. CABI Publishing, Wallingford, 6-8. https://doi.org/10.1079/9781786394118.0006

Mitsui H, van Achterberg K, Nordlander G, Kimura MT (2007) Geographical distributions and host associations of larval parasitoids of frugivorous Drosophilidae in Japan. Journal of Natural History 41: 1731-1738. https://doi.org/10.1080/00222930701504797

Nees von Esenbeck CG (1834) Hymenopterorum Ichneumonibus affinium monographiae, genera Europaea et species illustrantes, Stuttgartiae et Tubingae 1: 1-320. https://doi. org/10.5962/bhl.title.26555

Nomano FY, Kasuya N, Matsuura A, Suwito A, Mitsui H, Buffington ML, Kimura MT (2017) Genetic differentiation of Ganaspis brasiliensis (Hymenoptera: Figitidae) from East and Southeast Asia. Applied Entomology and Zoology 52: 429-437. https://doi.org/10.1007/ s13355-017-0493-0

Nomano FY, Mitsui H, Kimura MT (2015) Capacity of Japanese Asobara species (Hymenoptera; Braconidae) to parasitize a fruit pest Drosophila suzukii (Diptera; Drosophilidae). Journal of Applied Entomology 139: 105-113. https://doi.org/10.1111/jen.12141

Novković B, Mitsui H, Suwito A, Kimura MT (2011) Taxonomy and phylogeny of Leptopilina species (Hymenoptera: Cynipoidea: Figitidae) attacking frugivorous drosophilid flies in Japan, with description of three new species. Entomological Science 14: 333-346. https:// doi.org/10.1111/j.1479-8298.2011.00459.x

Noyes JS (2019) Universal Chalcidoidea Database. World Wide Web electronic publication. http://www.nhm.ac.uk/chalcidoids

Phillips DS (1993) Host-feeding and egg maturation by Pachycrepoideus vindemiae. Entomologia Experimentalis et Applicata 69: 75-82. https://doi.org/10.1111/j.1570-7458.1993. tb01730.x

Poyet M, Havard S, Prevost G, Chabrerie O, Doury G, Gibert P, Eslin P (2013) Resistance of Drosophila suzukii to the larval parasitoids Leptopilina heterotoma and Asobara japonica is 
related to haemocyte load. Physiological Entomology 38: 45-53. https://doi.org/10.1111/ phen. 12002

Ratnasingham S, Hebert PDN (2007) BOLD: The Barcode of Life Data System (www.barcodinglife.org). Molecular Ecology Notes 7: 355-364. https://doi.org/10.1111/j.14718286.2007.01678.x

Rosen P (1986) The role of taxonomy in effective biological control programs. Agriculture, Ecosystems, and the Environment 15: 121-129. https://doi.org/10.1016/01678809(86)90085-X

Rosen P, DeBach P (1973) Systematics, morphology, and biological control. Entomophaga 18: 215-222. https://doi.org/10.1007/BF02371468

Rossi Stacconi MV, Buffington M, Daane KM, Dalton DT, Grassi A, Kaçar G, Miller B, Miller JC, Baser N, Ioriatti C, Walton VM, Wiman NG, Wang X, Anfora G (2015) Host stage preference, efficacy and fecundity of parasitoids attacking Drosophila suzukii in newly invaded areas. Biological Control 84: 28-35. https://doi.org/10.1016/j.biocontrol.2015.02.003

Roy HE, Roy DB, Roques A (2011) Inventory of terrestrial alien arthropod predators and parasites established in Europe. BioControl 56: 477-504. https://doi.org/10.1007/s10526011-9355-9

Simon C, Frati F, Beckenbach A, Crespi B, Liu H, Flook P (1994) Evolution, weighting, and phylogenetic utility of mitochondrial gene sequences and a compilation of conserved polymerase chain reaction primers. Annals of the Entomological Society of America, 87L: 651-701. https://doi.org/10.1093/aesa/87.6.651

Stahl J, Tortorici F, Pontini M, Bon M-C, Hoelmer K, Marazzi C, Tavella L, Haye T (2019) First discovery of adventive populations of Trissolcus japonicus in Europe. Journal of Pest Science 92: 371-379. https://doi.org/10.1007/s10340-018-1061-2

Talamas EJ, Herlihy MV, Dieckhoff C, Hoelmer KA, Buffington M, Bon M-C, Weber DC (2015) Trissolcus japonicus (Ashmead) (Hymenoptera, Scelionidae) emerges in North America. Journal of Hymenoptera Research 43: 119-128. https://doi.org/10.3897/ JHR.43.4661

Thistlewood HMA, Gibson GAP, Gillespie DR, Fitzpatrick SM (2013) Chapter 21 - Drosophila suzukii (Matsumura), spotted wing drosophila. In: Mason PG, Gillespie DR (Eds) Biological Control Programmes in Canada 2001-2012, CABI Publishing, 152-155. https:// doi.org/10.1079/9781780642574.0152

Van Alphen JJM., Thunnissen I (1982) Host selection and sex allocation by Pachycrepoideus vindemiae Rondani (Pteromalidae) as a facultative hyperparasitoid of Asobara tabida Nees (Braconidae; Alysiinae) and Leptopilina heterotoma (Cynipoidea; Eucoilidae). Netherlands Journal of Zoology 33: 497-514. https://doi.org/10.1163/002829683X00228

Vet LEM, Janse C, van Achterberg C, van Alphen JJM (1984) Microhabitat location and niche segregation in two sibling species of Drosophilid parasitoids: Asobara tabida (Nees) and A. rufescens (Foerster) (Braconidae: Alysiinae). Oecologia 61: 182-188. https://doi. org/10.1007/BF00396757

Wang X-G, Kaçar G, Biondi A, Daane KM (2016) Foraging efficiency and outcomes of interactions of two pupal parasitoids attacking the invasive spotted wing drosophila. Biological Control 96: 64-71. https://doi.org/10.1016/j.biocontrol.2016.02.004 
Wang X-G, Nance AH, Jones JML, Hoelmer KA, Daane KM (2018) Aspects of the biology and reproductive strategy of two Asian larval parasitoids evaluated for classical biological control of Drosophila suzukii. Biological Control 121: 58-65. https://doi.org/10.1016/j. biocontrol.2018.02.010

Weber DC, Hajek AE, Hoelmer KA (2017) Chapter 1 - Accidental introductions of natural enemies: causes and implications. In: Proceedings of the $5^{\text {th }}$ International Symposium on the Biological Control of Arthropods (Ed.) CABI Publishing, Wallingford, 2-5. https:// doi.org/10.1079/9781786394118.0002

Wharton RA (1980) Review of the Nearctic Alysiini (Hymenoptera: Braconidae) with discussion of generic relationships within the tribe. University of California Publications in Entomology 88: 1-112.

Wharton RA (1997) Subfamily Alysiinae. In: Wharton RA, Marsh PM, Sharkey MJ (Eds) Manual of the New World Genera of the Family Braconidae (Hymenoptera). Special Publication No 1. International Society of Hymenopterists, Washington, DC, 84-116.

Wiman NG, Dalton DT, Anfora G, Biondi A, Chiu JC, Daane KM, Gerdeman B, Gottardello A, Hamby KA, Isaacs R, Grassi A, Ioriatti C, Lee JC, Miller B, Rossi Stacconi MV, Shearer PW, Tanigoshi L, Wang X, Walton VM (2016) Drosophila suzukii population response to environment and management strategies. Journal of Pest Science 89: 653-665. https://doi. org/10.1007/s10340-016-0757-4

Wiman NG, Walton VM, Dalton DT, Anfora G, Burrack HJ, Chiu JC, Daane KM, Grassi A, Miller B, Tochen S, Wang X, Ioriatti C (2014) Integrating temperature-dependent life table data into a matrix projection model for Drosophila suzukii population estimation. PLoS One 9: e106909. https://doi.org/10.1371/journal.pone.0106909

Yu DSK, van Achterberg C, Horstmann K (2016) Taxapad 2016 - World Ichneumonoidea 2015. Taxonomy, biology, morphology and distribution. USB flash drive. Nepean, Ontario. www.taxapad.com

\section{Supplementary material I}

Collection information for specimens reported in: New records of Leptopilina, Ganaspis, and Asobara species associated with Drosophila suzukii in North America, including detections of $L$. japonica and G. brasiliensis

Authors: Paul K. Abram, Audrey E. McPherson, Robert Kula, Tracy Hueppelsheuser, Jason Thiessen, Steve J. Perlman, Caitlin I. Curtis, Jessica L. Fraser, Jordan Tam, Juli Carrillo, Michael Gates, Sonja Scheffer, Matthew Lewis and Matthew Buffington

Data type: raw data

Copyright notice: This dataset is made available under the Open Database License (http://opendatacommons.org/licenses/odbl/1.0/). The Open Database License $(\mathrm{ODbL})$ is a license agreement intended to allow users to freely share, modify, and use this Dataset while maintaining this same freedom for others, provided that the original source and author(s) are credited.

Link: https://doi.org/10.3897/jhr.78.55026.suppl1 